\title{
A MURINE FEMORAL OSTECTOMY MODEL WITH HARDWARE EXCHANGE TO ASSESS ANTIBIOTIC-IMPREGNATED SPACERS FOR IMPLANT-ASSOCIATED OSTEOMYELITIS
}

\author{
R.P. Trombetta ${ }^{1,2}$, K.L. de Mesy Bentley ${ }^{2,3,4}$, E.M. Schwarz ${ }^{1,2,3,4}$, S.L. Kates ${ }^{2,5}$ and H.A. Awad ${ }^{1,2,4, *}$ \\ ${ }^{1}$ Department of Biomedical Engineering, University of Rochester, 207 Robert B. Goergen Hall, \\ Rochester, NY 14642, USA \\ ${ }^{2}$ Centre for Musculoskeletal Research, University of Rochester Medical Centre, \\ 601 Elmwood Avenue, Box 665, Rochester, NY 14642, USA \\ ${ }^{3}$ Department of Pathology and Laboratory Medicine, University of Rochester Medical Centre, \\ 601 Elmwood Avenue, Rochester, NY 14642, USA \\ ${ }^{4}$ Department of Orthopaedics, University of Rochester Medical Centre, 601 Elmwood Avenue, \\ Rochester, NY 14642, USA \\ ${ }^{5}$ Department of Orthopaedic Surgery, Virginia Commonwealth University School of Medicine, \\ Richmond, Virginia 23298, USA
}

\begin{abstract}
Implant-associated osteomyelitis is a chronic infection that complicates orthopaedic surgeries. Once infected, $50 \%$ of patients suffer treatment failure, resulting in high healthcare costs. While various small animal models have been developed to investigate the efficacy of prophylactic and therapeutic treatments, the minute scale of murine-model bone and hardware has been prohibitive for evaluating interventions with a complete implant exchange in the setting of an infected critical defect. To address this, the aim of the present study was to develop a murine femur model in which an initial mid-diaphyseal infection was established by surgical implantation of a titanium screw contaminated with bioluminescent Staphylococcus aureus (Xen36). $7 \mathrm{~d}$ after the infection was established, an ostectomy was performed to remove the middle segment ( $3 \mathrm{~mm}$ flanking the infected screw hole) and a bone-cement spacer, with or without impregnated gentamicin, was secured with a plate and screws to fix the septic segmental defect. Longitudinal bioluminescent imaging revealed a significant decrease in Xen36 growth following one-stage revision, with the antibiotic-impregnated spacer treated systemically with vancomycin $(p<0.05)$. This result was corroborated by a significant decrease in colony forming units (CFU) recovered from spacer, bone, soft tissue and hardware $12 \mathrm{~d}$ post-operative $(p<0.05)$. However, $\sim 10^{5} \mathrm{CFU} / \mathrm{g}$ Xen36 still persisted within the bone despite a clinical therapeutic regimen. Therefore, the model enables the investigation of new therapeutic strategies to improve upon the current standard of care in a mouse model of implant-associated osteomyelitis that employs reconstruction of a critical defect.
\end{abstract}

Keywords: Staphylococcus aureus, osteomyelitis, one-stage revision, poly(methyl methacrylate).

*Address for correspondence: Hani A. Awad, PhD, University of Rochester Medical Centre, 601 Elmwood Avenue, Box 665, Rochester, NY 14642, USA.

Telephone number: +1 5852735268 Email: hani_awad@urmc.rochester.edu

Copyright policy: This article is distributed in accordance with Creative Commons Attribution Licence (http://creativecommons.org/licenses/by-sa/4.0/).

\section{Introduction}

Orthopaedic surgery is plagued by implantassociated osteomyelitis. In the United States, the incidence rate of infected fractures with internal fixation is reported to be 7.8-23.6 \% (Colman et al., 2013; Morris et al., 2013; Momaya et al., 2016; Ruffolo et al., 2015). Yet, the occurrence rate of infection for cases pertaining to open fractures can be as high as $50 \%$ (Okike and Bhattacharyya, 2006). Rates of periprosthetic joint infections (PJIs) range around $0.54-1.41 \%$ and $0.5-0.92 \%$ for knee and hip, respectively (Gundtoft et al., 2015; Huotari et al., 2015; Lenguerrand et al., 2017). Once diagnosed, one out of two patients treated for implant-associated osteomyelitis suffers a subsequent failed treatment (Lee et al., 2010; Marculescu et al., 2006). Furthermore, the projected total costs in the US for PJI patients is 
expected to exceed $\$ 1.62$ billion by 2020 (Kurtz et al., 2012). Ultimately, osteomyelitis continues to be a clinical and socio-economic burden in the healthcare system despite the adherence to rigorous treatment algorithms that utilise prophylactic treatments, multiple revision surgeries and prolonged antibiotic regimens.

Staphylococcus aureus (S. aureus) is the most common pathogen in implant-associated osteomyelitis. This bacterial species accounts for $56 \%$ of all bone and joint infections (Grammatico-Guillon et al., 2012). The recalcitrant and recurring nature of chronic osteomyelitis is attributed to the ability of $S$. aureus to form biofilms on orthopaedic implants (Brady et al., 2006; Nishitani et al., 2015). Within the biofilm, S. aureus can resist the patient's immune response, as well as antibiotic therapies. Thus, effective management of the infection is difficult to achieve with the standard of care, consisting of intravenous antibiotics and highdose antibiotic therapy delivered after debridement to the infection site through vancomycin- and gentamicin-impregnated poly(methyl methacrylate) (PMMA) cement spacers (Del Pozo and Patel, 2009; Parvizi et al., 2009). Biofilm eradication requires an increased antibiotic concentration, up to 1000 times more than planktonic cells (Olson et al., 2002), and most standard-of-care antibiotics (e.g. vancomycin and gentamicin) are ineffective against bacteria in biofilms (Sanchez et al., 2015; van de Belt et al., 2000). Consequently, implant retention during staged revision surgery of established infections results in a $57 \%$ treatment failure rate (Lee et al., 2010; Marculescu et al., 2006). Implant retention is viewed as detrimental due to established biofilm on the hardware in delayed and late-onset osteomyelitis (Metsemakers et al., 2018). Thus, best-practice treatment algorithms recommend complete hardware exchange as the clinical standard when treating implant-associated chronic osteomyelitis. Yet, even with a removal strategy, a failure rate of $36 \%$ is still reported (Lee et al., 2010). Hence, alternative treatment strategies must be investigated to improve efficacy.

Small animal models of orthopaedic infection have investigated alternatives to the clinical standards in order to improve upon their shortcomings. Recently, clinically representative mouse models of one-stage revision (Yokogawa et al., 2018) have been established with bioluminescent $S$. aureus strains. Inzana et al. $(2015 a, b)$ created a septic defect model to investigate the local delivery of rifampin and vancomycin 3D-printed into calcium phosphate scaffolds. In this model, a mid-diaphyseal femoral defect was stabilised with plates and screws and contaminated with a methicillin-susceptible $S$. aureus (MSSA) collagen sheet. After $7 \mathrm{~d}$, the established infection was irrigated and debrided by a $3 \mathrm{~mm}$-long osteotomy, enabling insertion of antibiotic-laden spacers. However, this model retained plate stabilisation hardware during revision, which led to biofilm formation and persistence of bacteria (Yokogawa et al., 2018). Yokogawa et al. (2018) implanted a titanium femoral plate stabilised with two screws, one contaminated with methicillin-resistant $S$. aureus (MRSA). At time of revision, plate and screws were removed, screw holes reamed and new plates and screw implanted to investigate the synergy of systemic vancomycin and anti-glucosaminidase (Gmd) immunotherapy. Yet, this model lacks a cortical defect to provide local antibiotic therapy. To the authors knowledge, small animal models to perform hardware exchange in septic defects have not been developed, probably due to the technically daunting task of exchanging hardware without compromising the structural integrity of the bone (due to the minimal bone volume available for debridement). To overcome this challenge, a mouse model of implant-associated osteomyelitis that utilised a one-stage revision using complete hardware exchange combined with the insertion of an antibiotic-laden PMMA spacer was developed. This model aimed to mimic the clinical features of an implant-associated osteomyelitis and enabled the investigation of the clinically recommended treatment, including debridement, complete hardware exchange, systemic antibiotics and implantation of antibiotic spacers. Furthermore, this model could enable the investigation of new classes of antimicrobial-impregnated spacers, systemic antibiotics and additional novel therapeutics, such as antimicrobial-coated hardware. In the present validation study, one-stage revision with thorough debridement and complete hardware exchange was performed to compare two different cohorts of mice. One group of mice received only a spacer PMMA and acted as the negative control, while the other group received the clinical standard of treatment, gentamicin-laden PMMA with additional systemic antibiotics. The working hypothesis was that despite complete hardware exchange, the clinical standard treatment would fail to eradicate the bacteria on the implanted hardware, bone and soft tissue, enabling persistence of the infection.

\section{Materials and Methods}

\section{Study design}

For the development of the murine model, two groups were investigated: a negative control that received a PMMA spacer alone with no antibiotics and a clinically treated group that received both a gentamicin-laden PMMA spacer and additional daily systemic vancomycin $(110 \mathrm{mg} / \mathrm{kg}$ subcutaneously twice daily) (Caston et al., 2014). Vancomycin was allometrically scaled to simulate the pharmacokinetic profile used in clinical patients, receiving $1 \mathrm{~g}$ of vancomycin every $12 \mathrm{~h}$ (Albrecht et al., 1991; Crandon et al., 2010). The systemic vancomycin was administered for $12 \mathrm{~d}$ spanning the remainder of the study after the revision surgery. The negative control 
was used to observe the disease progression of the model with no therapeutic interventions, while the clinical control group was used to assess the efficacy of the scaled clinical standard of care used to treat the model.

During the initial infection surgery, a titanium screw contaminated with a bioluminescent strain of MSSA (Xen36) was inserted into the right femur of a 23-25-week-old female Balb/cJ mouse (Fig. 1). Prior to insertion, the screw was contaminated with Xen36 by submersion in bacterial culture for $20 \mathrm{~min}$ and, then, dried for $5 \mathrm{~min}$, resulting in a bacterial load of $\sim 2.5 \times 10^{6}$ colony forming units $(\mathrm{CFU}) / \mathrm{mL}$. Next, the infection was established over the time course of $7 \mathrm{~d}$ during which bioluminescent imaging (BLI) was performed at day 0, 1, 3, 5 and 7 to ensure equivalent progression of the infection. At day 7 , the revision surgery was performed, in which the contaminated titanium screw was removed and the femur was fixed with a polyether ether ketone (PEEK) plate with 4 titanium screws. A 3 mm-long ostectomy was performed to debride the infected mid-diaphysis of the femur. Then, a PMMA cement spacer with or without gentamicin (1.25 wt \%; Cobalt G-HV or MV; DJO Global, Vista, CA, USA) was inserted into the defect. Relapse in infection was continually monitored by BLI at day 1, 3, 5, 7 and $12 \mathrm{~d}$ post-revision. The mice were euthanised $12 \mathrm{~d}$ post-revision surgery and hardware, bone, cement spacer and surrounding soft tissue were harvested for CFU analysis (Fig. 1).

\section{Bioluminescent $S$. aureus strain}

Xen36 (PerkinElmer, Inc.) is a bioluminescent MSSA strain, which is a derivative of the clinical parental strain S. aureus ATCC 49525 (Wright) harbouring a stable copy of the luxABCDE operon. This strain was chosen due to its proven high bioluminescent signal in vivo relative to other bioluminescent $S$. aureus strains, as well as its use in other murine models of implant-associated osteomyelitis (Farnsworth et al., 2015; Inzana et al., 2015a; Pribaz et al., 2012).

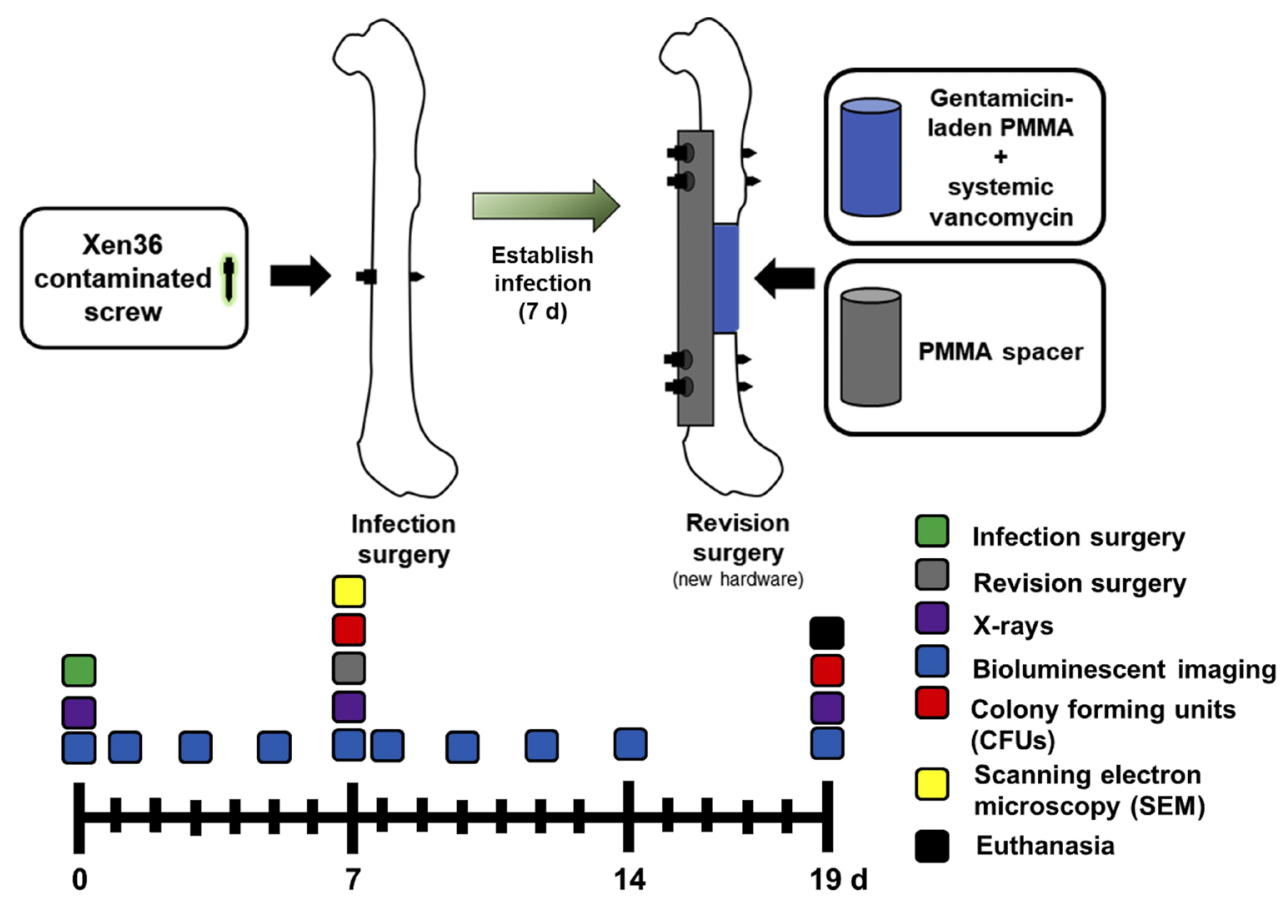

Fig. 1. Schematic summary of the implant-associated osteomyelitis mouse model employing a hardware exchange revision. During the initial infection surgery, a titanium screw contaminated with a bioluminescent strain of MSSA (Xen36) was inserted into the right femur of a 23-25-week-old female Balb/cJ mouse. Prior to insertion, the screw was contaminated with Xen36 by submersion in bacterial culture for 20 min and, then, dried for $5 \mathrm{~min}$, resulting in a bacterial load of $\sim 2.5 \times 10^{6} \mathrm{CFU} / \mathrm{mL}$. Next, the infection was established over the time course of $7 \mathrm{~d}$ during which BLI was performed at day $0,1,3,5$ and 7 to ensure equivalent progression of infection. At day 7, the revision surgery was performed, in which the contaminated titanium screw was removed and the femur was fixed with a PEEK plate with 4 titanium screws. A 3-mm-long ostectomy was performed to debride the infected mid-diaphysis of the femur. CFU analysis of the removed screw and debrided bone determined bacterial counts of $1.5 \times 10^{5} \mathrm{CFU} / \mathrm{g}$ and $4.0 \times 10^{7} \mathrm{CFU} / \mathrm{g}$, respectively. Then, a PMMA cement spacer with or without gentamicin (1.25 wt \%; Cobalt G-HV or MV; DJO Global) was inserted into the defect. Relapse in infection was continually monitored through BLI at day 1, 3, 5, 7 and $12 \mathrm{~d}$ post-revision. Mice were euthanised $12 \mathrm{~d}$ post-revision surgery and hardware, bone, cement spacer and surrounding soft tissue were harvested for CFU analysis. Two groups were investigated, a standardof-care group, that received local delivery of gentamicin through PMMA spacers and systemic antibiotics that began immediately after revision surgery $(110 \mathrm{mg} / \mathrm{kg}$ vancomycin injected subcutaneously twice daily), and a negative control that received no antibiotics (both locally and systemically). 


\section{Contamination of titanium screw with bioluminescent $S$. aureus}

A single colony of Xen 36 was used to prepare bacteria cultures grown at $37^{\circ} \mathrm{C}, 225 \mathrm{rpm}$ in tryptic soy broth (TSB) until the optical density $\left(\mathrm{OD}_{630 \mathrm{~nm}}\right)$ of $0.4-0.5$ was reached (log phase), corresponding to $\sim 1.5 \times 10^{\circ} \mathrm{CFU} /$ $\mathrm{mL}$. Subsequently, $200 \mu \mathrm{L}$ of the bacterial cultures were transferred to a 96-well microtiter plate (Falcon; Corning Life Sciences). Then, a titanium screw (Shenzhen Qianpeng Weiye Technology Co., Ltd, Shenzen, China) was contaminated by submersion in the plate for $20 \mathrm{~min}$ and allowed to dry for $5 \mathrm{~min}$ prior to implantation. The bacterial load on each titanium screw prior to implantation was determined to be $2.475 \times 10^{6}\left( \pm 1.49 \times 10^{6}\right) \mathrm{CFU} / \mathrm{mL}$ by sonicating adhered bacteria and plating serial dilutions on TSB agar.

\section{Animals and inoculation surgery}

All animal procedures were performed in accordance to protocols approved by the University of Rochester's Committee on Animal Resources (UCAR protocol 2013-001). Female Balb/cJ mice, 13-15 weeks old, were purchased from Jackson Research Labs. Mice were housed until surgeries were performed, at around $23-25$ weeks of age. $0.1 \mathrm{mg} / \mathrm{kg}$ buprenorphine was subcutaneously administered pre-operatively and twice daily for $3 \mathrm{~d}$ following each surgery. Throughout the 12-week time course of the study, no differences in body weights were observed between the two treatment groups (data not shown).

Briefly, an inoculation surgery was performed with a contaminated titanium screw to establish an implant-associated infection. Prior to surgeries, mice were anaesthetised with $12 \mathrm{mg} / \mathrm{kg}$ xylazine and $130 \mathrm{mg} / \mathrm{kg}$ ketamine through intraperitoneal injection. The right femur of the mouse was exposed by a direct lateral approach using blunt dissection to reveal the femur. A titanium screw contaminated with Xen36 was inserted into the mid-diaphysis of the femur after drilling through the anterolateral and posterolateral cortices using a $0.31 \mathrm{~mm}$ drill bit (Fig. $2 \mathbf{a}, \mathbf{b})$. Then, muscle and skin were closed using 5-0 monofilament nylon sutures and the infection was allowed to establish for $7 \mathrm{~d}$.

\section{Revision surgery}

After the infection was established, a revision surgery was performed, in which the contaminated screw was removed, the infected area was irrigated, the femur was fixed, the visible pathological tissue was debrided, the infected bone was ostectomised and
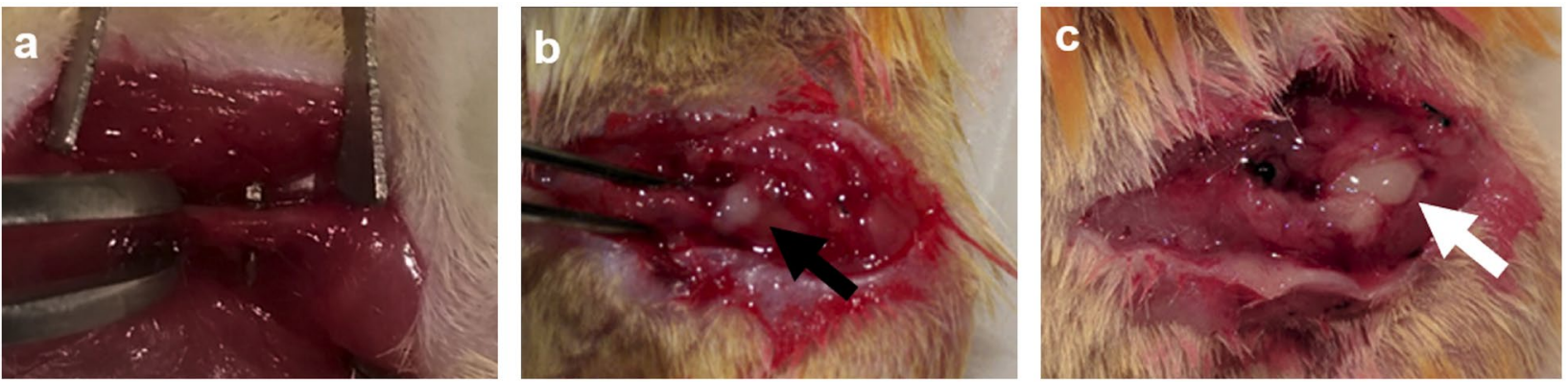

Fig. 2. Macroscopic evidence of infection $7 \mathrm{~d}$ post-inoculation surgery. (a) A representative photograph of a mouse's femur exposed using blunt dissection prior to mid-diaphysis implantation of a titanium screw contaminated with Xen36 during the inoculation surgery is shown (day 0). Gross examination of the established infection at the time of revision (day 7 post-infection) generally showed (b) peri-implant abscesses (black arrow) and (c) subcutaneous abscesses (white arrow). At this time, the quantified viable bacteria on the screw were determined to be $\sim 7.4 \times 10^{5} \mathrm{CFU}$.
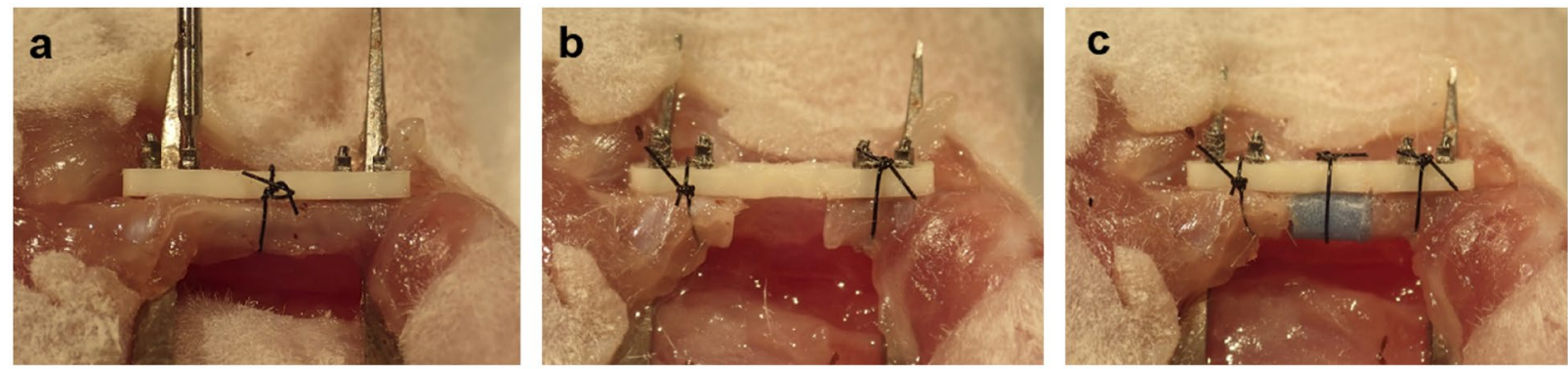

Fig. 3. Insertion of a cement spacer was achieved by aggressive debridement of the mid-diaphysis by performing a 3-mm-long ostectomy. (a) After the contaminated screw was removed, the infection site was irrigated and a PEEK fracture fixation plate was fixed to the anterolateral surface of the femur with 4 titanium screws. (b) Radical debridement was performed ( $25 \%$ of the length of the femur) by attaching a cutting guide to the fixation plate and screws and using a Gigli wire to cut a $3 \mathrm{~mm}$-long defect. (c) A PMMA spacer was inserted into the defect and secured in cerclage fashion with a braided suture. 
an antibiotic spacer was inserted (Fig. 3a-c). Briefly, mice were anaesthetised with $12 \mathrm{mg} / \mathrm{kg}$ xylazine and $130 \mathrm{mg} / \mathrm{kg}$ ketamine injected intraperitoneally. The wound was reopened and any abscess or pathological tissue was debrided. The femur was re-exposed and the contaminated titanium screw was removed and either placed in $2.5 \%$ glutaraldehyde/4\% paraformaldehyde in $0.1 \mathrm{M}$ sodium cacodylate buffer for scanning electron microscopy (SEM) analysis or frozen in $300 \mu \mathrm{L}$ of phosphate buffered saline (PBS) at $-80{ }^{\circ} \mathrm{C}$ for later $\mathrm{CFU}$ analysis. After removal of the screw, the femur was irrigated and, then, fixed on the anterolateral surface with a 6-hole radiolucent PEEK plate and 4 titanium screws inserted into the outermost screw holes (Shenzhen Qianpeng Weiye Technology Co., Ltd, Shenzen, China). The PEEK plate was used to enable longitudinal micro-computed tomography imaging without the production of metallic artefacts for future studies examining and quantifying bone healing. Next, a 5-0 nylon monofilament suture was tied around the plate and femur in between the proximal and distal pairs of screws, in order to prevent screw pull-out from possible septic loosening. A $3 \mathrm{~mm}$-long transverse ostectomy encompassing the location of the original infected screw insertion was performed at the mid-diaphysis by using a $0.67 \mathrm{~mm}$ wire Gigli saw and cutting guide, allowing fixed and reproducible cuts (RISystem, Davos, Switzerland). The $3 \mathrm{~mm}$ long bone debrided segment was placed in $1 \mathrm{~mL}$ of PBS and stored at $-80{ }^{\circ} \mathrm{C}$ for CFU analysis. The fixed and ostectomised femur was again irrigated. Commercial PMMA bone cement spacers, with (Cobalt G-HV, DJO Global) and without 1.25 wt \% gentamicin (Cobalt MV, DJO Global), were prepared in accordance to the manufacturer's guidelines and shaped into $1.8 \times 3 \mathrm{~mm}$ cylindrical spacers using a custom negative mould machined out of aluminium to fit these dimensions. The cured PMMA spacers were inserted into the femoral defect and secured in a cerclage fashion using a 6-0 nylon braided suture (Fig. 3b,c). Finally, muscle and skin were closed and the mice underwent a treatment time of $12 \mathrm{~d}$ postrevision surgery. The sample size was 6 mice per group. When performing the surgical procedures, three mice died during revisions, two suffered femoral fractures and the other did not recover from anaesthesia. These outcomes were associated with the learning curve for this surgical technique. These mice were replaced to maintain sample size. Additionally, no mice died during the study; however, one mouse had to be removed from the PMMA-treated group due to a fractured femur after osteolysis.

\section{Radiographic imaging}

To qualitatively assess implant placement and bone changes, X-rays were taken at 0, 7 (before and after revision surgery) and $12 \mathrm{~d}$ post-revision. An X-ray cabinet (LX-60 X-ray Cabinet, Faxitron Bioptics LCC, Tucson, AZ, USA) was used to take X-rays at $26 \mathrm{kV}$, with an exposure time of $6 \mathrm{~s}$. Mice were anaesthetised and placed in the supine position to capture an anteroposterior X-ray of a lateral view of the operated femur.

\section{SEM}

SEM (LEO 982 FE-SEM, Carl Zeiss SMT, Thornwood, NY, USA) of gold-sputtered titanium screws was used for qualitative assessment of $S$. aureus adhered to the surface of the screw after removal of the screw $7 \mathrm{~d}$ post-infection surgery, as previously described (Varrone et al., 2014). The surface of the hardware was carefully scanned at $2000 \times$ to identify rounded structures. S. aureus can appear as 2 dividing bacteria or in planktonic aggregates (Nishitani et al., 2015). Once a rounded structure was found, the magnification was increased to $5000 \times$ for closer morphologic assessment and verification of the structure size (1.0 to $1.25 \mu \mathrm{m})$.

\section{Longitudinal BLI}

BLI of the mice infected with Xen36 was performed at day $0,1,3,5,7,8,10,12,14$ and 19 using a Spectrum imaging system (IVIS; PerkinElmer, Inc.). Ventral images at each time point were taken with automatic high sensitivity exposure settings. The bioluminescence was quantified using the LivingImage software 2.50.1 (PerkinElmer, Inc.) by analysing a fixed region of interest (ROI) around the infected femur of each mouse. The ROI was designated to fit around the infected femur of each mouse, encompassing the entire BLI signal. The bioluminescence was quantified as average radiant efficiency ( $\left.\mathrm{p} / \mathrm{s} / \mathrm{cm}^{2} / \mathrm{sr}\right)$.

\section{Quantifying bacterial colonisation of harvested tissue and implants}

At the end of the time course, mice were sacrificed by carbon dioxide inhalation followed by cervical dislocation. Hardware, spacer, bone and soft tissue (quadricep and hamstring muscle blocks) were harvested to quantify the viable bacteria load for each sample. The PMMA cement spacer, fixation hardware and screw harvested at revision surgery were sonicated in $1 \mathrm{~mL}$ of sterile PBS for $3 \mathrm{~min}$ at $35 \mathrm{kHz}$ (VWR Symphony Ultrasonic Cleaner VWR Intl., Radnor, PA, USA) and vortexed to release any adhered bacteria. Bone, soft tissue and ostectomised bone at revision surgery were homogenised in $3 \mathrm{~mL}$ of sterile PBS using a T10 basic ULTRA-TURRAX ${ }^{\circledR}$ disperser (IKA Works, Inc., Wilmington, NC, USA). Then, the homogenised and sonicated solutions were plated on TSB agar in 10-fold serial dilutions and incubated overnight at $37^{\circ} \mathrm{C}$ to count for CFU the next day. CFU counts were normalised to the mass of each collected sample and transformed using $\log _{10}(1+X)$ before statistical analysis.

\section{Statistical analysis}

Longitudinal body weight and bioluminescence differences were assessed by performing a multiplicity adjusted two-way ANOVA. A log 
transformation of the bioluminescence data was performed prior to performing the two-way ANOVA, which was followed by Sidak's test for multiple comparisons. The means of the area under the curve of the bioluminescence data was compared by performing an unpaired student $t$-test. Bacterial CFU quantification was tested for Gaussian distribution by performing a Kolmogorov-Smirnov test with DallalWilkinson-Lillie for corrected $p$-value. The failure in the normality test resulted in data being analysed by Mann-Whitney nonparametric $t$-test. In addition, CFU data were binarised and Fisher's exact test was performed on the $2 \times 2$ contingency table. Samples were categorised as infected versus uninfected by defining uninfected samples by the lowest CFU count. All differences were considered significant for $p<0.05$. All data were represented as mean, except for bacterial CFU quantification, which was represented as median due to the bimodal distribution. Statistical analyses were performed using GraphPad Prism 6 (GraphPad Software, La Jolla, CA, USA).

\section{Results}

Similar levels of infection in the two groups were achieved from the inoculation surgery as assessed at day 7 by four independent outcomes. First, occurrences of subcutaneous and peri-implant abscesses were observed in infected mice during the revision surgery (Fig. 2b,c). Also, no difference in CFU were found on the inoculation screw $\left[1.22 \times 10^{4} \pm 4.60 \times 10^{3} \mathrm{CFU}\right.$ for PMMA and $1.85 \times 10^{4} \pm 1.22 \times 10^{4} \mathrm{CFU}$ for gentamicinPMMA + SC Vanco (systemic vancomycin); $p=0.3054$ by Mann-Whitney U test] and in the ostectomy $\left(2.63 \times 10^{7} \pm 8.69 \times 10^{6} \mathrm{CFU} / \mathrm{g}\right.$ for PMMA and $5.14 \times 10^{7} \pm 6.47 \times 10^{7}$ for gentamicin-PMMA + SC Vanco; $p=0.9697$ by Mann-Whitney U test) harvested during revision surgery at day 7. Furthermore, SEM showed similar levels of bacterial colonisation on the recovered screws, observing the presence of S. aureus cells located on the screw as well as embedded within a biofilm (Fig. 4a-c).

BLI of the infected mice (Fig. 5i) confirmed the lack of statistically significant differences in the levels of bacterial presence in both groups after the inoculation surgery ( $p>0.05$ by 2 -way ANOVA). Consistent with similar murine models (Inzana et al., 2015a; Inzana et al., 2015b; Yokogawa et al., 2018), BLI peaked at day $3\left(\sim 3.5 \times 10^{4}\right.$ photons $\left./ \mathrm{s} / \mathrm{cm}^{2} / \mathrm{sr}\right)$ and declined to $\sim 9.3 \times 10^{3}$ photons $/ \mathrm{s} / \mathrm{cm}^{2} / \mathrm{sr}$ prior to revision surgery at day 7. Longitudinal BLI also detected relapse of the infection in the PMMA-only group, which peak between day 1 and 3 of the revision surgery (day 8-10 post inoculation surgery). In contrast, gentamicin-PMMA + SC Vanco significantly reduced the BLI levels to background levels following revision surgery ( $p<0.001$ by 2 -way ANOVA).

Radiographic imaging revealed no osteolysis in any mice prior to revision surgery. However, at the end of the experiment, radiographic evidence of peri-implant osteolysis was present in all the mice in the PMMA-only group. In contrast, there was no radiographic evidence of osteolysis in any of the mice in the gentamicin-PMMA + SC Vanco group (Fig. 6ah).

To further validate this observation, bone, soft tissue, cement spacer and hardware were harvested at the end time point and either homogenised or sonicated and serially diluted for bacterial enumeration and CFU plating. At the time of harvest, all cement spacers remained intact in the defect (Fig. 6). CFU counts demonstrated a significant reduction in bacterial colonisation for each of the 4 harvest samples between PMMA and gentamicin-PMMA + SC Vanco groups, resulting in 0.9-, 1.7-, 0.9- and 3.1-log reductions for bone, soft tissue, hardware and cement spacer, respectively (Fig. 7a-d). This finding corroborated the longitudinal bioluminescent measurements.

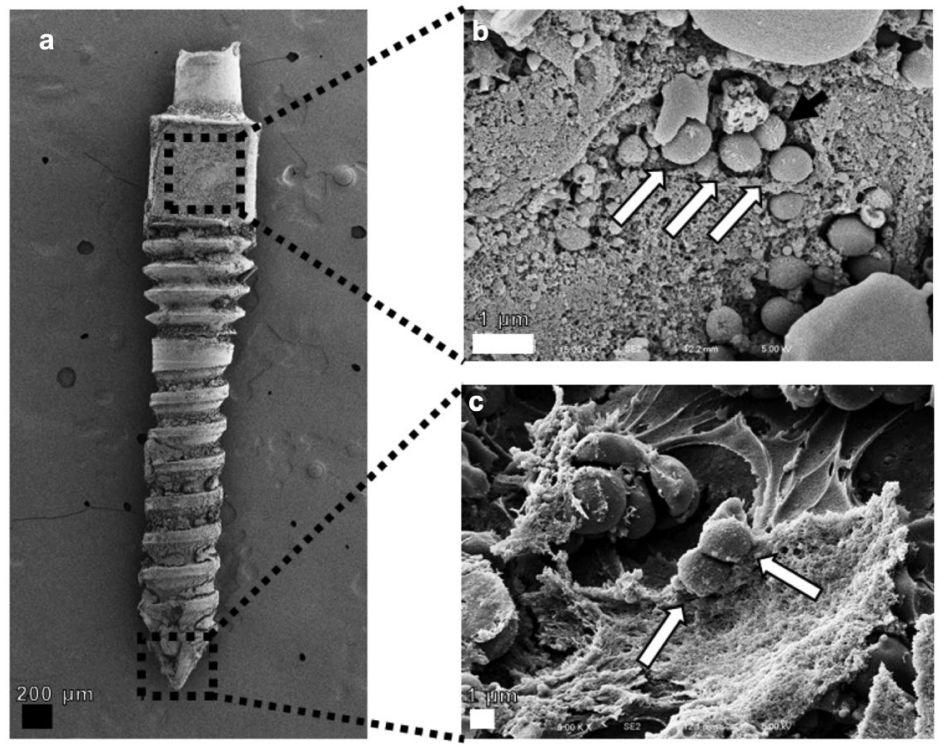

드닌요
Fig. 4. Bacterial colonisation of the titanium screw $7 \mathbf{d}$ after infection surgery. Representative SEM images of an explanted screw $7 \mathrm{~d}$ post-inoculation surgery obtained initially at nominal instrument magnifications of (a) $\times 25$, (b) $\times 15,000$ and (c) $\times 5,000$. (a) $S$. aureus cells were located throughout the infected screw. (b) S. aureus cells located at the head of the screw (white arrows), as well as the tip of the screw (c; white arrows) embedded within biofilm. (c) Cluster of cocci (arrows), which was indicative of $S$. aureus proliferation within the biofilm on the implant. 

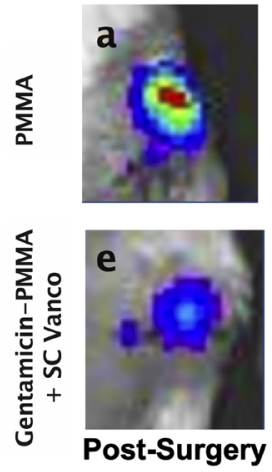

i
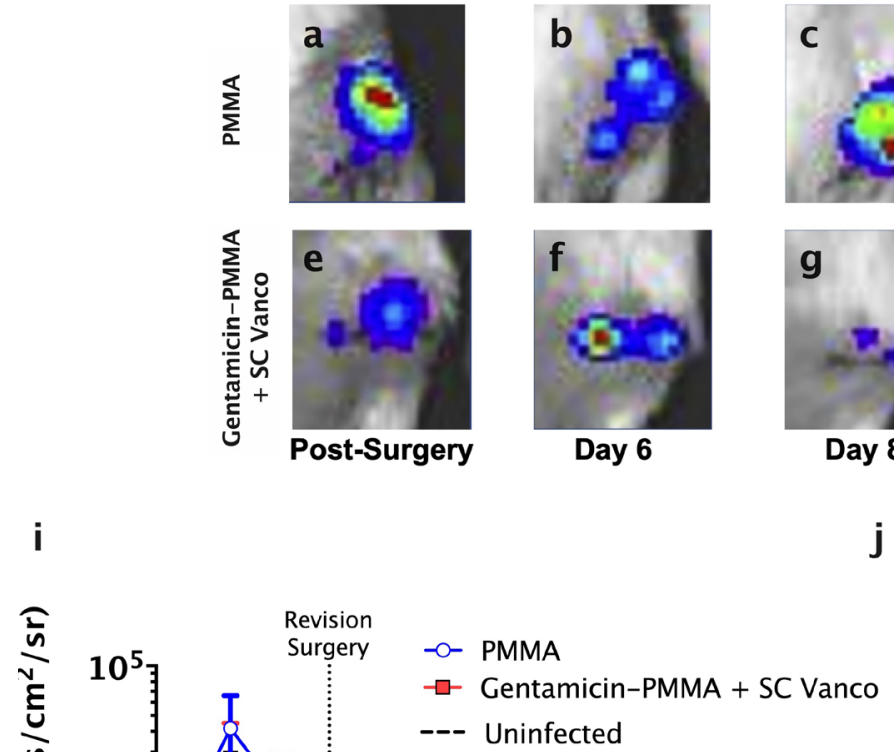

Day 6
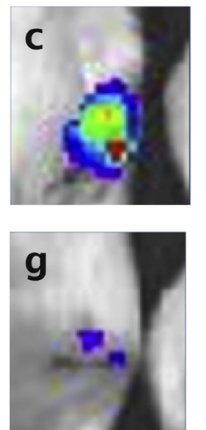

Day 8
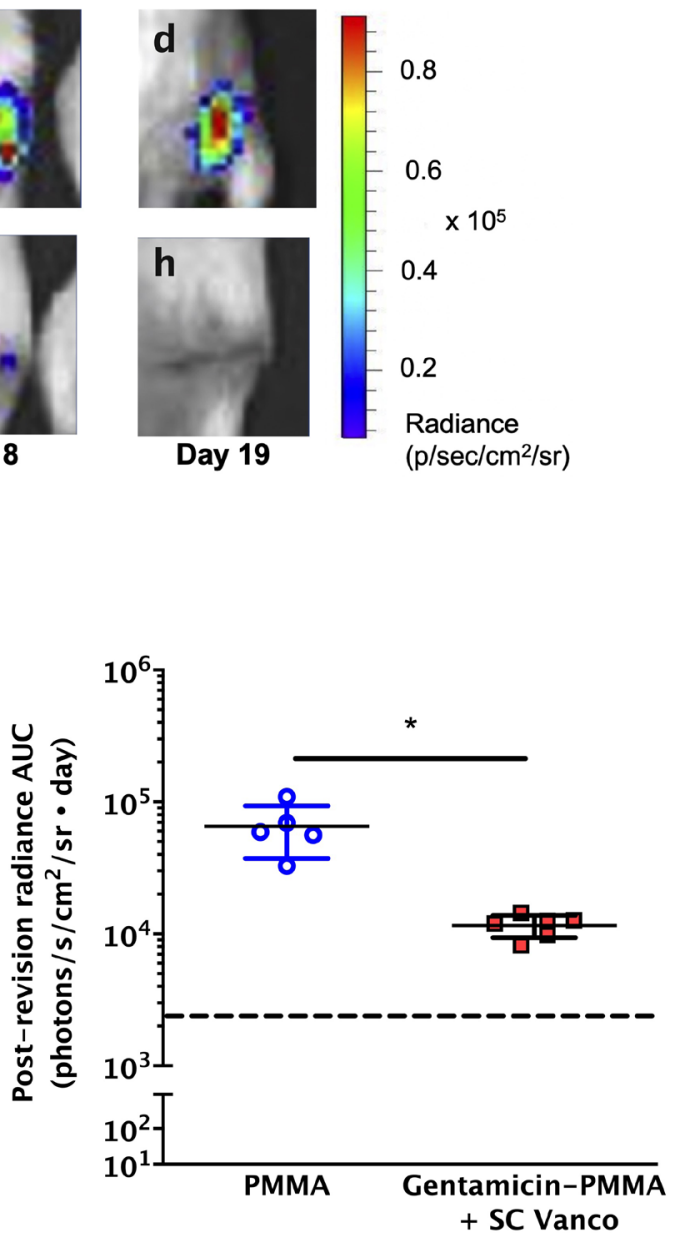

Fig. 5. Local and systemic delivery of antibiotics significantly reduced the bacterial burden following revision, as measured by BLI. After revision surgery, mice were treated with either PMMA spacers alone or PMMA spacers laden with gentamicin and additional systemic (SC) vancomycin. Relapse of the infection was assessed by BLI imaging 1, 3, 5, 7 and $12 \mathrm{~d}$ post-revision surgery. Representative longitudinal BLI images of (a-d) PMMA and (e-h) PMMA-laden with gentamicin- and SC-vancomycin-treated mice are shown. (i) Quantitative BLI data demonstrated that treatment with gentamicin-laden PMMA and SC vancomycin significantly reduced the bacterial burden 1,3,5 and $7 \mathrm{~d}$ post-revision surgery. (j) A significant reduction in bacterial burden was observed for mice treated with the clinical standard in comparison to the PMMA-only group, as determined by computing the area under the curve post-revision surgery. ${ }^{*}<0.05$ vs PMMA-only after 2-way ANOVA with Sidak's test for multiple comparisons. \# $p<0.05$ by unpaired student $t$-test. AUC (?) data presented as a scatter plot with mean and standard deviation. Dashed line represents baseline BLI as measured in sterile mice. $n=6$ for gentamicin-PMMA + SC Vanco; $n=5$ for PMMA.

\section{Discussion}

Implant-associated osteomyelitis is a clinical challenge due to its recurring and unpredictable nature. Complications and failure rates remain high, despite well-established surgical principles (Crockarell et al., 1998; Schoifet and Morrey, 1990; Teeny et al., 1990). To better understand clinical outcomes and potential therapeutic avenues, a clinically representative mouse model of established implant-associated osteomyelitis was developed, modelling the first step in a two-stage revision. The present work expanded upon several established murine models designed to evaluate interventions for established implant-related osteomyelitis (Reizner et al., 2014).
The most common mouse models are periprosthetic models of infection, which are employed to investigate the efficacy of different treatment modalities in a posttotal knee joint arthroplasty infection (Bernthal et al., 2010; Berretta et al., 2017; Kaur et al., 2016; Niska et al., 2012; Pribaz et al., 2012; Stavrakis et al., 2016; Thompson et al., 2017; Young et al., 2015). In these models, the inoculation strategy generally consists of directly injecting $10^{2}-10^{4} \mathrm{CFU}$ of $S$. aureus into the joint space after that an orthopaedic hardware, typically a k-wire, has been implanted in a retrograde fashion. Investigated treatment strategies for this model generally consist of systemic antibiotics (Thompson et al., 2017), antimicrobial-coated hardware (Bernthal et al., 2010; Stavrakis et al., 2016), antibiotic-laden pastes (Berretta et al., 2017) or prophylactic treatments 
(Niska et al., 2012; Young et al., 2015). However, these models have limited relevance, because they do not include hardware exchange.

Mouse models of implant-associated osteomyelitis have also been developed using internal fixation. Traditionally, internal fixation has been performed in mouse models with either a transcortical pin or an intramedullary implant that has been previously infected (Li et al., 2008; Matsuno et al., 2006; Shandley et al., 2012; Varoga et al., 2008). However, these models lack the ability to perform a revision surgery in order to debride the infected bone and insert an antibiotic-laden spacer, which is a common practice for trauma-related cases. In order to circumvent this, a model utilising a locking fracture fixation plate was developed to study the efficacy of bone debridement and the utilisation of antibiotic-laden cement spacers (Inzana et al., 2015a). In this model, an infection is established in a mid-diaphyseal femoral ostectomy through implantation of a contaminated sponge. Then, a revision surgery is performed $7 \mathrm{~d}$ postinfection, in which debridement of the infected bone is accomplished by widening the ostectomy to $3 \mathrm{~mm}$, a partial exchange of the hardware is performed and an antibiotic-laden spacer is inserted to assess infection management. Although this model has some relevance, such as displaying the bone infection hallmarks of chronic osteomyelitis, the retention of the hardware is inconsistent with most revision surgeries.

A similar mouse osteomyelitis model established an infection by using an contaminated screw for fixation of a femoral plate to investigate the synergy between systemic vancomycin treatment with anti-glucosaminidase monoclonal antibodies (antiGmd) immunotherapy (Yokogawa et al., 2018). This model replicates common clinical practices in which the hardware is exchanged during the one-stage revision surgery. However, this model does not contain a cortical defect to allow for structural bone healing outcomes. Additionally, while the study demonstrated synergistic effects between antibiotic and immunotherapy, resulting in reduced bacterial burden and osseointegration of the new implants, $\sim 10^{3} \mathrm{CFU} / \mathrm{mL}$ were still recovered on the newly exchanged hardware. This indicated the need for more radical debridement and local delivery of antibiotics in addition to systemic antibiotics in order to better manage the infection.

The current study presented a novel murine model of implant-associated osteomyelitis, which utilised a one-stage revision surgery for removal of the infected hardware, debridement of the infected ROI and introduction of a new sterile hardware for femoral fixation of a critical defect with local antibiotic delivery. In this model, a bioluminescent strain of MSSA, Xen36, was chosen in order to longitudinally monitor the progression of the infection in vivo. The bioluminescent signal peaked $3 \mathrm{~d}$ post-infection surgery, which was similar both in timing and magnitude to other models of implantassociated osteomyelitis that utilise bioluminescent MSSA (Inzana et al., 2015a; Inzana et al., 2015b; Li et al., 2008; Yokogawa et al., 2018). The revision surgery resulted in a reduction in bioluminescence, that quickly rebounded after $24 \mathrm{~h}$ for the untreated control PMMA group. The infection was established with an inserted titanium screw that had a diameter of $\sim 0.3 \mathrm{~mm}$, yet despite a radical $3 \mathrm{~mm}$ ostectomy ( $\sim 25 \%$ of the femur), the infection still quickly rebounded. This highlighted the ability of the deep infection to spread to adjacent tissues and prevail in seemingly healthy tissue despite debridement and irrigation of the identified devitalised and infected tissues. However, the clinical standard of gentamicinimpregnated PMMA spacer with systemic antibiotics displayed no such rebound due to the administration of local and systemic antibiotics. The observed decline in signal after day 3 prior to the revision surgery, which was also seen in the group of mice that received PMMA after revision, was attributed to the transition from planktonic bacterial growth (high metabolic

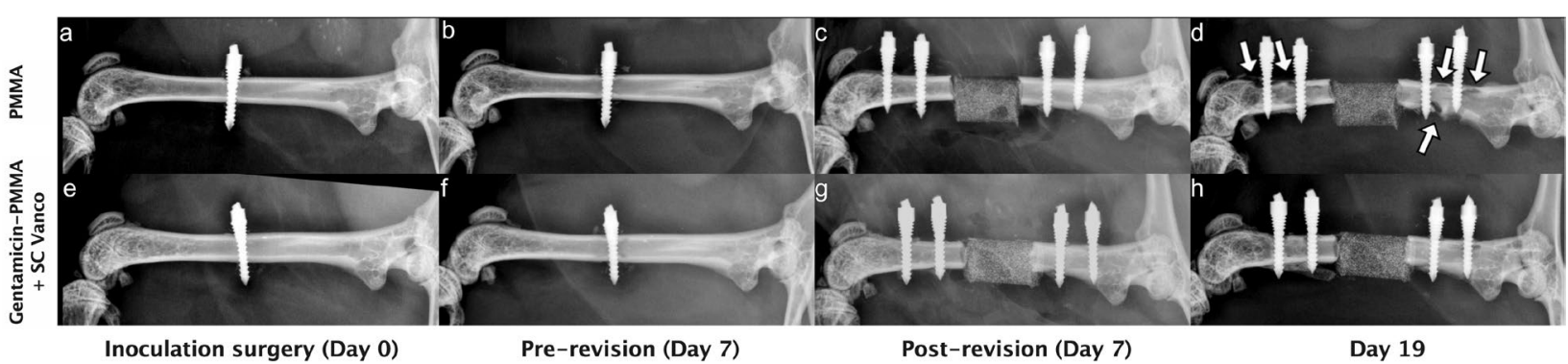

Fig. 6. Local and systemic antibiotic therapy prevented peri-implant osteolysis following revision surgery. (a,e) A contaminated titanium screw with Xen36 was inserted into the mid-diaphysis of a mouse's femur. $(\mathbf{b}, \mathbf{f})$ The infection was allowed to establish for $7 \mathrm{~d}$, in which a revision surgery was performed. No osteolysis was observed prior to revision surgery. For revision, the contaminated screw was removed and a new hardware was inserted. $(\mathbf{c}, \mathbf{g})$ Debridement of the mid-diaphysis was performed resulting in a $3 \mathrm{~mm}-\mathrm{long}$ defect in which a bone cement spacer with or without gentamicin was inserted. (d) Radiographic evidence of osteolysis was apparent by day 19 in the PMMA-only group (white arrows). (h) No osteolysis was observed in the clinical treatment group; PMMA spacer with gentamicin and SC vancomycin. Representative X-rays for each treatment group are all from the same mouse. 

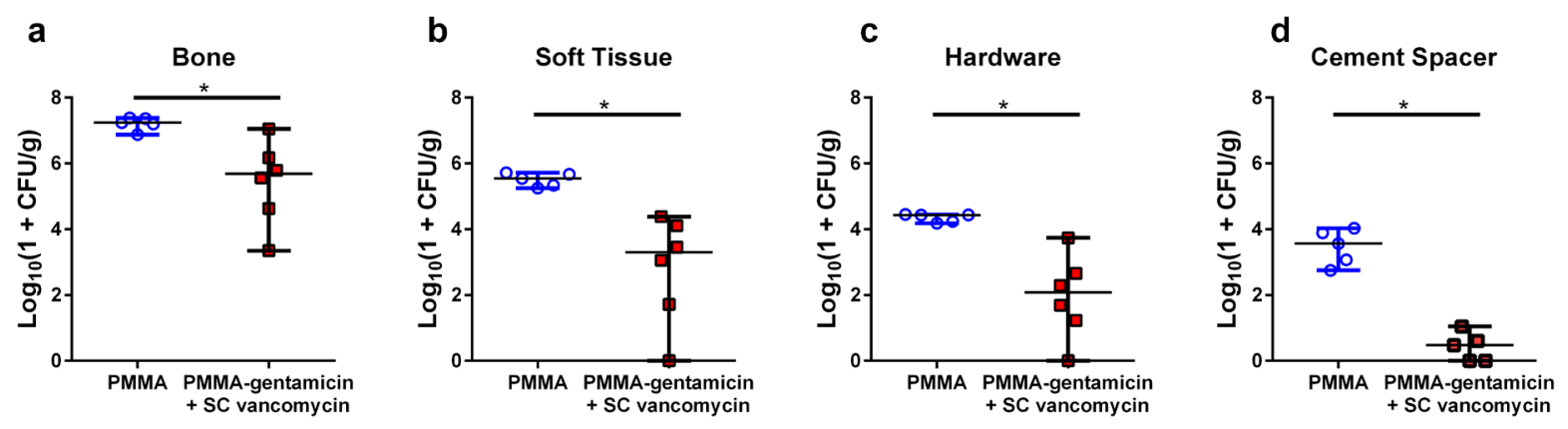

Fig. 7. Local and systemic antibiotic therapy decreased bacterial burden following revision surgery. $12 \mathrm{~d}$ after the revision surgery, mice were euthanised and bone, soft tissue, hardware and cement spacer were harvested for quantification of bacterial colonisation. CFU analysis demonstrated that the clinical standard of care, PMMA cement impregnated with gentamicin and systemic antibiotics, had a significant reduction in recovered viable bacteria by (a) 0.9-log for harvested bone, (b) 1.7-log for soft tissue, (c) 0.9-log for hardware and (d) 3.1-log for cement spacer, relative to the PMMA-only control. ${ }^{*} p<0.05$ between groups as determined by the Mann-Whitney $t$-test for nonparametric data. $n=6$ for gentamicin-PMMA +SC Vanco; $n=5$ for PMMA. Data presented as scatter plots with median and $95 \%$ confidence interval for each group.

rate) to colonised bacteria growth (low metabolic rate) (Li et al., 2008), biofilm formation (Inzana et al., 2015b), host's innate immune response and ability of planktonic S. aureus cells to become localised within both macrophages and osteoblasts, while still surviving (Hamza and Li, 2014; Josse et al., 2015), and to propagate into the osteocytic-canalicular networks (de Mesy Bentley et al., 2017; de Mesy Bentley et al., 2018).

Treating the established infection with the clinical standard of care enabled the bacterial burden to be significantly reduced after the revision surgery in comparison to the PMMA-only-treated mice (Fig. 5i,j). The clinically treated mice displayed a bioluminescent signal that drifted just above the sterile control post-revision (Fig. 5i), no osteolysis in longitudinal radiographs (Fig. 6) and a benign skin suture surface. Thus, qualitative signs of infection were not apparent in the clinically treated group. However, recovered bacteria in both groups indicated the inability to sufficiently clear the infection even with the antibiotic treatment. Although the clinical treatment significantly reduced the bacterial count in hardware, spacer, bone and soft tissue, only minimal $\log$ reductions were observed and none were completely sterile (Fig. 7a-d). Furthermore, Fisher's exact test did not yield significance when calculating the ability to eradicate the infection for the recovered hardware, spacer, bone and soft tissue. This indicated that even when managed with a standard clinical treatment, the infection was not eradicated, which is analogous to the persistence of infection observed clinically. Thus, the new described model provided the capacity to investigate novel therapeutics that could be delivered locally to improve upon current standards.

The ability of the bacteria to persist highlights the elusive nature of chronic osteomyelitis, especially with the absence of clinical signs (Mouzopoulos et al., 2011; Panteli and Giannoudis, 2016). Explanations as to why the reinfection rates of osteomyelitis are as high as $20-30 \%$ (Conterno and Turchi, 2013), even with debridement beyond the margins, is still poorly understood. However, one explanation is the ability of $S$. aureus to propagate into the bone canaliculi to evade therapeutic regimens and host immune responses and, thus, emerging after revision surgery with debridement and insertion of a new hardware (de Mesy Bentley et al., 2017). Hence, the presented model provided a platform to investigate experimental treatments consisting of new classes of spacers and antimicrobials that could improve upon therapeutic outcomes even with thorough debridement.

The model of implant-associated osteomyelitis with one-stage hardware exchange was not without limitations. First, to perform the ostectomy of the mid-diaphysis after implant removal and irrigation, the femur had to be fixed prior to debridement. In a clinical setting, complete debridement and irrigation would be carried out prior to the implantation of a new hardware. However, because of the limitations imposed on surgical procedures by the small size of the murine bone, the femur was first fixed and then the ostectomy was performed. This was required for the surgical procedure because a cutting guide was attached to the fixation hardware in order to perform consistent osteotomies mouse to mouse. Secondly, the infection surgery did not represent a truly realistic scenario when a single titanium screw would be implanted. Similar models have utilised screws, pins and k-wires to establish an infection (Choe et al., 2015; Li et al., 2008; Pribaz et al., 2012). However, this limitation was abated by the clinically relevant revision surgery used to treat the established infection. Lastly, the limitations of using a small 
animal model must also be considered in the context of assessing local drug delivery. This impacted the effective drug diffusion distance needed for effective treatment, which could bias the clinical interpretation of the strength of delivered drugs.

\section{Conclusion}

A mouse model of a one-stage revision surgery for implant-associated osteomyelitis was developed, demonstrating the limitations of antimicrobial PMMA spacers. This model could serve as a platform to examine novel therapeutics for osteomyelitis in future studies.

\section{Acknowledgements}

The authors are grateful for the technical assistance and expertise of Gayle Schneider of the electron microscope shared resource at the University of Rochester Medical Centre. The authors would also like to thank Noriaki Yokogawa for his insightful discussions on orthopaedic treatment strategies. This study was supported by the NIAMS/NIH grants P30AR069655 and P50AR072000 and the AOTrauma Clinical Priority Program.

\section{References}

Albrecht LM, Rybak MJ, Warbasse LH, Edwards DJ (1991) Vancomycin protein binding in patients with infections caused by Staphylococcus aureus. DICP 25: 713-715.

Bernthal NM, Stavrakis AI, Billi F, Cho JS, Kremen TJ, Simon SI, Cheung AL, Finerman GA, Lieberman JR, Adams JS, Miller LS (2010) A mouse model of postarthroplasty Staphylococcus aureus joint infection to evaluate in vivo the efficacy of antimicrobial implant coatings. PLoS One 5: e12580. DOI: 10.1371/journal. pone.0012580.

Berretta JM, Jennings JA, Courtney HS, Beenken KE, Smeltzer MS, Haggard WO (2017) Blended chitosan paste for infection prevention: preliminary and preclinical evaluations. Clin Orthop Relat Res 475: 1857-1870.

Brady RA, Leid JG, Costerton JW, Shirtliff ME (2006) Osteomyelitis: clinical overview and mechanisms of infection persistence. Clin Microbiol Newslett 28: 65-72.

Caston JJ, Gonzalez-Gasca F, Porras L, Illescas S, Romero MD, Gijon J (2014) High vancomycin minimum inhibitory concentration is associated with poor outcome in patients with methicillin-susceptible Staphylococcus aureus bacteremia regardless of treatment. Scand J Infect Dis 46: 783-786.

Choe H, Narayanan AS, Gandhi DA, Weinberg A, Marcus RE, Lee Z, Bonomo RA, Greenfield EM (2015) Immunomodulatory peptide IDR-1018 decreases implant infection and preserves osseointegration. Clin Orthop Relat Res 473: 2898-2907.

Colman M, Wright A, Gruen G, Siska P, Pape H-C, Tarkin I (2013) Prolonged operative time increases infection rate in tibial plateau fractures. Injury 44 : 249-252.

Conterno LO, Turchi MD (2013) Antibiotics for treating chronic osteomyelitis in adults. Cochrane Database Syst Rev: CD004439. DOI: 10.1002/14651858. CD004439.pub3.

Crandon JL, Kuti JL, Nicolau DP (2010) Comparative efficacies of human simulated exposures of telavancin and vancomycin against methicillinresistant Staphylococcus aureus with a range of vancomycin mics in a murine pneumonia model. Antimicrob Agents Chemother 54: 5115-5119.

Crockarell JR, Hanssen AD, Osmon DR, Morrey BF (1998) Treatment of infection with debridement and retention of the components following hip arthroplasty. J Bone Joint Surg Am 80: 1306-1313.

de Mesy Bentley KL, MacDonald A, Schwarz EM, Oh I (2018) Chronic osteomyelitis with Staphylococcus aureus deformation in submicron canaliculi of osteocytes: a case report. JBJS Case Connect 8: e8. DOI: 10.2106/JBJS.CC.17.00154.

de Mesy Bentley KL, Trombetta R, Nishitani K, Bello-Irizarry SN, Ninomiya M, Zhang L, Chung HL, McGrath JL, Daiss JL, Awad HA, Kates SL, Schwarz EM (2017) Evidence of Staphylococcus aureus deformation, proliferation, and migration in canaliculi of live cortical bone in murine models of osteomyelitis. J Bone Miner Res 32: 985-990.

Del Pozo JL, Patel R (2009) Infection associated with prosthetic joints. N Engl J Med 361: 787-794.

Farnsworth CW, Shehatou CT, Maynard R, Nishitani K, Kates SL, Zuscik MJ, Schwarz EM, Daiss JL, Mooney RA (2015) A humoral immune defect distinguishes the response to Staphylococcus aureus infections in mice with obesity and type 2 diabetes from that in mice with type 1 diabetes. Infect Immun 83: 2264-2274. DOI: 10.1128/IAI.03074-14.

Grammatico-Guillon L, Baron S, Gettner S, Lecuyer AI, Gaborit C, Rosset P, Rusch E, Bernard L (2012) Bone and joint infections in hospitalized patients in France, 2008: clinical and economic outcomes. J Hosp Infect 82: 40-48.

Gundtoft PH, Overgaard S, Schønheyder HC, Møller JK, Kjærsgaard-Andersen P, Pedersen AB (2015) The "true" incidence of surgically treated deep prosthetic joint infection after 32,896 primary total hip arthroplasties: a prospective cohort study. Acta Orthop 86: 326-334.

Hamza T, Li B (2014) Differential responses of osteoblasts and macrophages upon Staphylococcus aureus infection. BMC Microbiology 14: 207-207.

Huotari K, Peltola M, Jämsen E (2015) The incidence of late prosthetic joint infections. Acta Orthop 86: 321-325.

Inzana JA, Schwarz EM, Kates SL, Awad HA (2015a) A novel murine model of established Staphylococcal bone infection in the presence of a 
fracture fixation plate to study therapies utilizing antibiotic-laden spacers after revision surgery. Bone 72: 128-136.

Inzana JA, Trombetta RP, Schwarz EM, Kates SL, Awad HA (2015b) 3D printed bioceramics for dual antibiotic delivery to treat implant-associated bone infection. Eur Cell Mater 30: 232-247.

Josse J, Velard F, Gangloff SC (2015) Staphylococcus aureus vs. osteoblast: relationship and consequences in osteomyelitis. Front Cell Infect Microbiol 5: 85. DOI: 10.3389/fcimb.2015.00085.

Kaur S, Harjai K, Chhibber S (2016) In vivo assessment of phage and linezolid based implant coatings for treatment of methicillin resistant $S$. aureus (MRSA) mediated orthopaedic device related infections. PLoS One 11: e0157626. DOI: 10.3389/ fcimb.2015.00085.

Kurtz SM, Lau E, Watson H, Schmier JK, Parvizi J (2012) Economic burden of periprosthetic joint infection in the United States. J Arthroplasty 27: 6165.e61. DOI: 10.1016/j.arth.2012.02.022.

Lee J, Kang CI, Lee JH, Joung M, Moon S, Wi YM, Chung DR, Ha CW, Song JH, Peck KR (2010) Risk factors for treatment failure in patients with prosthetic joint infections. J Hosp Infect 75: 273-276.

Lenguerrand E, Whitehouse M, Beswick A, Jones S, Porter M, Blom A (2017) Increasing burden of infection and risk of early revision following hip and knee replacement: evidence from the National Joint Registry for England and Wales. Bone Joint Res 6: 391-398. doi: 10.1302/2046-3758.66.BJR-2017-0003.R1.

Li D, Gromov K, Søballe K, Puzas JE, O'Keefe RJ, Awad H, Drissi H, Schwarz EM (2008) A Quantitative mouse model of implant-associated osteomyelitis and the kinetics of microbial growth, osteolysis and humoral immunity. J Orthop Res 26: 96-105.

Marculescu CE, Berbari EF, Hanssen AD, Steckelberg JM, Harmsen SW, Mandrekar JN, Osmon DR (2006) Outcome of prosthetic joint infections treated with debridement and retention of components. Clin Infect Dis 42: 471-478.

Matsuno H, Yudoh K, Hashimoto M, Himeda Y, Miyoshi T, Yoshida K, Kano S (2006) A new antibacterial carrier of hyaluronic acid gel. J Orthop Sci 11: 497-504.

Metsemakers WJ, Kuehl R, Moriarty TF, Richards RG, Verhofstad MHJ, Borens O, Kates S, Morgenstern M (2018) Infection after fracture fixation: current surgical and microbiological concepts. Injury 49: 511-522.

Momaya AM, Hlavacek J, Etier B, Johannesmeyer D, Oladeji LO, Niemeier TE, Herrera N, Lowe JA (2016) Risk factors for infection after operative fixation of tibial plateau fractures. Injury 47: 15011505.

Morris BJ, Unger RZ, Archer KR, Mathis SL, Perdue AM, Obremskey WT (2013) Risk factors of infection after ORIF of bicondylar tibial plateau fractures. J Orthop Trauma 27: e196-200.

Mouzopoulos G, Kanakaris NK, Kontakis G, Obakponovwe O, Townsend R, Giannoudis PV
(2011) Management of bone infections in adults: the surgeon's and microbiologist's perspectives. Injury 42 Suppl 5: S18-23.

Nishitani K, Sutipornpalangkul W, de Mesy Bentley KL, Varrone JJ, Bello-Irizarry SN, Ito H, Matsuda S, Kates SL, Daiss JL, Schwarz EM (2015) Quantifying the natural history of biofilm formation in vivo during the establishment of chronic implantassociated Staphylococcus aureus osteomyelitis in mice to identify critical pathogen and host factors. J Orthop Res 33: 1311-1319.

Niska JA, Shahbazian JH, Ramos RI, Pribaz JR, Billi F, Francis KP, Miller LS (2012) Daptomycin and tigecycline have broader effective dose ranges than vancomycin as prophylaxis against a Staphylococcus aureus surgical implant infection in mice. Antimicrob Agents Chemother 56: 2590-2597.

Okike K, Bhattacharyya T (2006) Trends in the management of open fractures: a critical analysis. JBJS 88: 2739-2748.

Olson ME, Ceri H, Morck DW, Buret AG, Read RR (2002) Biofilm bacteria: formation and comparative susceptibility to antibiotics. Can J Vet Res 66: 86-92.

Panteli M, Giannoudis PV (2016) Chronic osteomyelitis: what the surgeon needs to know. EFORT Open Reviews 1: 128-135.

Parvizi J, Azzam K, Ghanem E, Austin MS, Rothman RH (2009) Periprosthetic infection due to resistant staphylococci: serious problems on the horizon. Clin Orthop Relat Res 467: 1732-1739.

Pribaz JR, Bernthal NM, Billi F, Cho JS, Ramos RI, Guo Y, Cheung AL, Francis KP, Miller LS (2012) Mouse model of chronic post-arthroplasty infection: noninvasive in vivo bioluminescence imaging to monitor bacterial burden for long-term study. J Orthop Res 30: 335-340.

Reizner W, Hunter JG, O'Malley NT, Southgate RD, Schwarz EM, Kates SL (2014) A systematic review of animal models for Staphylococcus aureus osteomyelitis. Eur Cell Mater 27: 196-212.

Ruffolo MR, Gettys FK, Montijo HE, Seymour RB, Karunakar MA (2015) Complications of high-energy bicondylar tibial plateau fractures treated with dual plating through 2 incisions. J Orthop Trauma 29: 8590.

Sanchez CJ, Jr., Shiels SM, Tennent DJ, Hardy SK, Murray CK, Wenke JC (2015) Rifamycin derivatives are effective against staphylococcal biofilms in vitro and elutable from PMMA. Clin Orthop Relat Res 473: 2874-2884.

Schoifet SD, Morrey BF (1990) Treatment of infection after total knee arthroplasty by debridement with retention of the components. J Bone Joint Surg Am 72: 1383-1390.

Shandley S, Matthews KP, Cox J, Romano D, Abplanalp A, Kalns J (2012) Hyperbaric oxygen therapy in a mouse model of implant-associated osteomyelitis. J Orthop Res 30: 203-208.

Stavrakis AI, Zhu S, Hegde V, Loftin AH, Ashbaugh AG, Niska JA, Miller LS, Segura T, Bernthal NM (2016) In vivo efficacy of a "smart" 
antimicrobial implant coating. J Bone Joint Surg Am 98: 1183-1189.

Teeny SM, Dorr L, Murata G, Conaty P (1990) Treatment of infected total knee arthroplasty. Irrigation and debridement versus two-stage reimplantation. J Arthroplasty 5: 35-39.

Thompson JM, Saini V, Ashbaugh AG, Miller RJ, Ordonez AA, Ortines RV, Wang Y, Sterling RS, Jain SK, Miller LS (2017) Oral-only linezolid-rifampin is highly effective compared with other antibiotics for periprosthetic joint infection: study of a mouse model. J Bone Joint Surg Am 99: 656-665.

van de Belt $H$, Neut D, Schenk W, van Horn JR, van der Mei HC, Busscher HJ (2000) Gentamicin release from polymethylmethacrylate bone cements and Staphylococcus aureus biofilm formation. Acta Orthop Scand 71: 625-629.

Varoga D, Tohidnezhad M, Paulsen F, Wruck CJ, Brandenburg L, Mentlein R, Lippross S, Hassenpflug J, Besch L, Müller M, Jürgens C, Seekamp A, Schmitt L, Pufe T (2008) The role of human $\beta$-defensin-2 in bone. J Anat 213: 749-757.

Varrone JJ, de Mesy Bentley KL, Bello-Irizarry SN, Nishitani K, Mack S, Hunter JG, Kates SL, Daiss JL, Schwarz EM (2014) Passive immunization with anti-glucosaminidase monoclonal antibodies protects mice from implant-associated osteomyelitis by mediating opsonophagocytosis of Staphylococcus aureus megaclusters. J Orthop Res 32: 1389-1396.

Yokogawa N, Ishikawa M, Nishitani K, Beck CA, Tsuchiya H, Mesfin A, Kates SL, Daiss JL, Xie C, Schwarz EM (2018) Immunotherapy synergizes with debridement and antibiotic therapy in a murine onestage exchange model of MRSA implant-associated osteomyelitis. J Orthop Res 36: 1590-1598.

Young SW, Roberts T, Johnson S, Dalton JP, Coleman B, Wiles S (2015) Regional intraosseous administration of prophylactic antibiotics is more effective than systemic administration in a mouse model of TKA. Clin Orthop Relat Res 473: 3573-3584.

\section{Discussion with Reviewers}

Reviewer 2: In clinical practice, patients are often taken off antibiotics for a few days to reduce the risk of false negatives from cultured biopsies. In the present study, animals received antibiotic therapy up to the day of euthanasia. Could this influence the results of the study?

Authors: We understand the merit of having a washout period and how in an ideal scenario this would enable no presence of antibiotics to confound the resulting bacterial counts. However, we believe this is not an issue in the present study because all mice, except for one, still had bacterial colonisation in bone, soft tissue and hardware samples. In the presence of consistent culture negative samples, this would warrant a washout period to ensure antibiotics were not present during CFU plating.
Yet, it is important to consider that a washout period in a preclinical model would also enable any surviving persister cells or biofilm to rebound, causing an observed increase in virulence as well as increased CFU that no longer would correspond to the true quantified measure at time of halting the therapeutic treatment. This would additionally skew any bacterial counts higher.

The fact that CFU were observed and counted in all but one sample for bone, soft tissue and hardware confirmed the recalcitrant nature of implant-associated osteomyelitis, which was the study original hypothesis.

Reviewer 2: Vancomycin is not a first-choice antibiotic for treatment of MSSA (with possible exception of patients with penicillin allergy). Knowing that vancomycin is a weakly bactericidal, could alternative, first choice antibiotic regimens improve treatment outcome?

Authors: Vancomycin may be used with MSSA infections for patients who have penicillin allergies. Also, other antibiotics may be used as well. But herein lies an issue with the treatment of osteomyelitis, where there is no consensus amongst literature or clinicians on a doctrine of antibiotics to be used in certain scenarios with various broad-spectrum antibiotics, various strains of $S$. aureus and the increasing rise of antibiotic resistance.

Reviewer 2: How do you interpret the failed treatment in the presented model? If clinical goldstandard has a good treatment success rate, why does the model have no treatment success? Could it be due to debridement issues or the chosen antibiotic regimen?

Authors: No mouse was found to be absent of bacterial colonisation after the time course of the study. For soft tissue, cement spacer and hardware, 1,2 and 1 sample(s), respectively, were culturenegative, while all other samples were culturepositive. This highlighted the difficulty in complete eradication of bacterial colonisation using clinically relevant therapeutics, which has also been observed in a hardware retention model in which vancomycinladen PMMA does not enable infection-free mice after the time course of the study (Inzana et al., 2015b). Although reduction in bacterial counts is possible, complete eradication of bacteria is a daunting task for clinician, especially with the finding that $S$. aureus can infiltrate into the bone canalicular network, offering a further explanation for recurrence of infection and recovered bacteria in harvested bone samples.

Ceylan Windolf: Why PEEK plates were used instead of titanium plates, as done for the screws?

Authors: PEEK plates were used over titanium plates to eliminate the metallic artefacts that titanium plates produce during micro-computed tomography imaging. This was necessary to accurately quantify bone healing within the ostectomised defect. 
Willemijn Boot: Why was vancomycin chosen in this model for treatment of a MSSA infection?

Authors: Vancomycin is commonly employed as a systemic antibiotic for MSSA infections, especially for those patients who have a penicillin allergy. Furthermore, previous mouse models and treatments have administered systemic vancomycin for MSSA infections (Inzana et al., 2015b; Wang et al., 2006, additional reference). Additionally, no appreciable differences in minimum inhibitory concentration (MIC) are observed between MRSA and MSSA for vancomycin.

Scot Guelcher: Why was a group with PMMA + subcutaneous Vanco (no gentamicin) not included? Authors: A PMMA + SC Vanco group has similar measured outcomes (BLI and recovered CFU) to that of a PMMA-antibiotics (i.e. Vanco) + SC antibiotic group (Inzana et al., 2015a; Inzana et al., 2015b). Additionally, in aseptic defect models representing trauma cases, antibiotic-laden bone cement will be inserted, while PMMA absent of antibiotics will be not. Therefore, the inclusion of PMMA + SC Vanco only in the current study was waived.

\section{Additional Reference}

Wang G, Hindler JF, Ward KW, Bruckner DA (2006) Increased vancomycin MICs for Staphylococcus aureus clinical isolates from a university hospital during a 5-year period. J Clin Microbiol 44: 3883-3886.

Editor's note: The Scientific Editor responsible for this paper was Fintan Moriarty. 